\title{
CHANGES IN MAGNETIC ANISOTROPY OF MO FILMS INDUCED BY NITROGEN DIFFUSION FROM PROTECTIVE LAYERS
}

\author{
M. Kratzer, S. Becker, H. Rohrmann and K. Röll \\ Universität Gh Kassel, Exp. Physik IV, 34109 Kassel, Germany
}

Abstract - RE/TM films used for magneto-optical (MO) data storage are commonly covered with reactively sputtered SiN protective layers. For stoichiometric $\mathrm{Si}_{3} \mathrm{~N}_{4}$ cover layers no reaction between the magnetic and the protective layer is expected. But if there is a surplus of nitrogen within the protective layer the magnetic properties and the long-term stability of the magnetic layer may be influenced.

To investigate this we prepared ferrimagnetic TbFeCo-films covered with a SiN protective layer containing a surplus of nitrogen. For testing their long-term stability the films were annealed several times. To analyze the magnetic properties measurements of Kerr hysteresis were performed. The magnetic anisotropy was measured by Kerr Torque Magnetometry, a very sensitive magneto-optical method which provides local information about the anisotropy field at the surface region of the magnetic layer.

As a result substantial changes of the magnetic properties at the surface region of the TbFeCo layers were detected. These changes were induced by a diffusion of nitrogen from the protective into the magnetic layer leading to a reduced content of magnetically active $\mathrm{Tb}$ at the surface. At longer annealing times this led in extreme to a double layer behaviour which makes the medium useless for MO data storage.

KEYWORDS: KERR MEASUREMENT; KERR TORQUE MAGNETOMETRY; LONG-TERM STABILITY; PROTECTIVE LAYER; MAGNETIC ANISOTROPY; RE/TM-FILMS

\section{INTRODUCTION}

To protect the magneto-optical (MO) layer from oxidation and to enhance the readout signal, RE/TM films used in the magneto-optical storage technology are commonly covered with reactively sputtered SiN films.

For stoichiometric $\mathrm{Si}_{3} \mathrm{~N}_{4}$ cover layers no reaction between the magnetic and the protective layer is expected [1]. On the other hand it is known, that nitrogen affects the magnetic properties of ferrimagnetic TbFeCo films substantially due to a reaction between $\mathrm{Tb}$ and nitrogen. This had been observed if nitrogen was mixed to the sputtering gas during the deposition of the ferrimagnetic TbFeCo films [2].

During the reactive sputtering of SiN, however, often an excess of nitrogen is applied in the sputtering atmosphere. The sputtering process then leads to the preparation of a SiN layer with a surplus of nitrogen, because in dependence on the $\mathrm{N}_{2}$ fraction in the Ar- $\mathrm{N}_{2}$ sputtering atmosphere the composition of the SiN film can be varied from a nitrogen rich to a nitrogen deficient state [3].

Therefore, the nitrogen excess within the SiN layer may also influence the properties of the magneto-optical film at the interface between the magnetic and the dielectric layer. As a result this process may affect the long-term stability of the MO recording media considerably.

To investigate this we examined $\mathrm{TbFeCo}$ films covered with SiN protective layers that were sputtered with a surplus of nitrogen. To detect possible changes within the magnetic layer we used measurements of Kerr hysteresis and the Kerr Torque Magnetometry $[4,5]$.

\section{EXPERIMENTAL SETUP}

For data acquisition the polar Kerr effect was measured by a Kerr magnetometer (fig. 1) using the Faraday modulator technique [6]. The measurements of Kerr hysteresis could be performed in a temperature range from room temperature up to $550 \mathrm{~K}$.

To perform the Kerr torque measurements the magnetic field with a maximum amplitude of $\mathrm{B}_{\max }=2,3 \mathrm{~T}$ could be applied in any direction $\alpha$ by rotating the magnet. Since the Kerr signal $\theta_{k}(H, \alpha)$ is proportional to the out of plane component $M_{\perp}$ the equilibrium angle $\theta$ of the magnetization can be determined directly from the Kerr data (fig.2). This is quite in contrast to the conventional torque magnetometry, where the angle $\theta$ is unknown. 


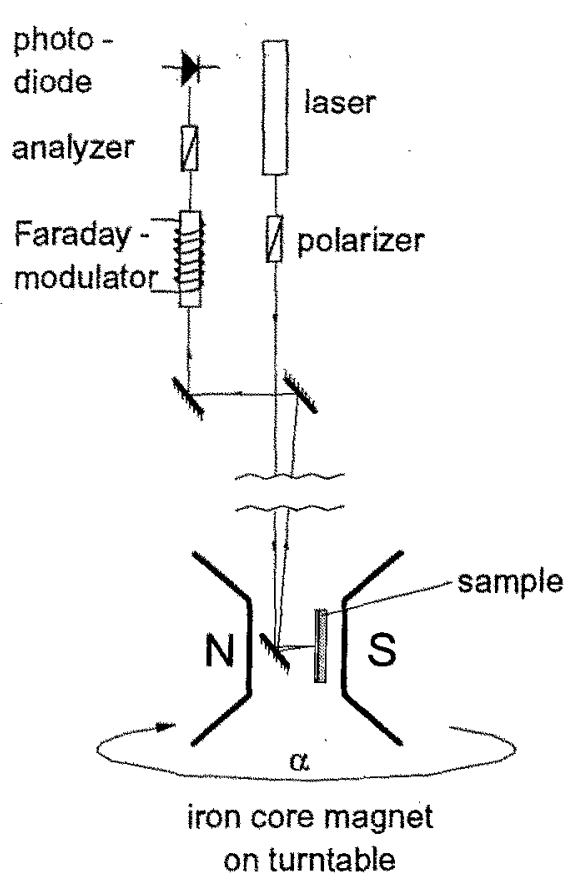

fig. I: Design of the Kerr magnetometer

For the further analysis of the Kerr data we use a common approach for the anisotropy energy of the magnetic layer consisting of quadratic and bi-quadratic terms

$$
\varepsilon_{k}(\theta)=K_{1} \cdot \sin ^{2} \theta+K_{2} \cdot \sin ^{4} \theta
$$

The calculation with the procedure of Kerr Torque Magnetometry then provides the quadratic $\left(2 \mathrm{~K}_{1} / \mathrm{M}_{\mathrm{s}}=\right.$ $\left.\mu_{0} H_{k 1}\right)$ and bi-quadratic $\left(2 \mathrm{~K}_{2} / \mathrm{M}_{\mathrm{s}}=\mu_{\mathrm{o}} \mathrm{H}_{\mathrm{k} 2}\right)$ terms of the anisotropy field within the information depth of the laser beam [7].

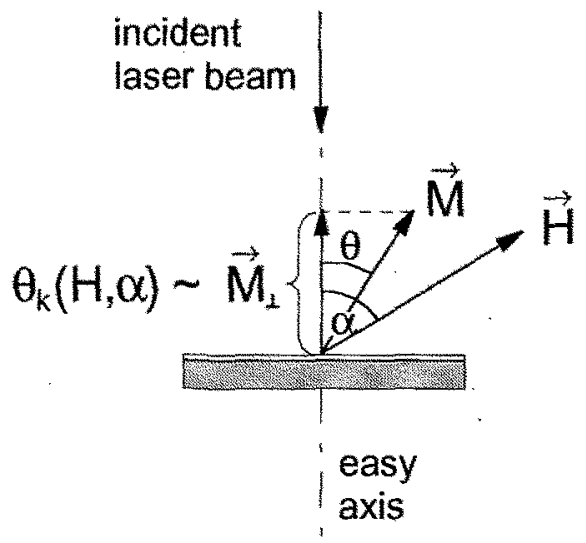

fig. 2: Determination of the equilibrim angle $\theta$ of the magnetization by measurement of the polar Kerr effect.

\section{EXPERIMENTS AND RESULTS}

To investigate a possible reaction between the magnetic and the protective layer ferrimagnetic $\mathrm{Tb}_{32} \mathrm{Fe}_{60} \mathrm{Co}_{8}$ films with a thickness of 100 to $500 \mathrm{~nm}$ were sputtered which are dominated magnetically by the RE content. The layers were prepared from a mosaic target and were embedded between two $\mathrm{SiN}$ layers that were reactively sputtered with a surplus of nitrogen (fig. 3 ).

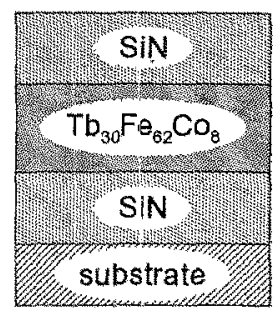

fig. 3: Cross section of the samples covered with a dielectric SiN layer containing a surplus of nitrogen.

The samples were annealed several times for typically one hour at $500 \mathrm{~K}$ up to a total time of $15 \mathrm{~h}$. After every step of annealing the Kerr hysteresis and Kerr torque measurements were carried out at room temperature. As an example fig. 4 shows the results for a sample with a thickness of $500 \mathrm{~nm}$. In the as deposited state the sample shows a Kerr hysteresis which is typical for a reversal process in direction of the easy axis. The Kerr torque measurement provides an almost perfect perpendicular uniaxial anisotropy because of a very small bi-quadratic term $\mu_{\mathrm{o}} \mathrm{H}_{\mathrm{k} 2}$.

After annealing for $1 \frac{1}{2} \mathrm{~h}$ there are no significant changes visible in the Kerr hysteresis. But, quite in contrast, substantial changes can be seen using the Kerr Torque Magnetometry. The bi-quadratic term $\mu_{\mathrm{o}} \mathrm{H}_{\mathrm{k} 2}$ that can be assigned to an inhomogeneity of the anisotropy within the probed volume [8] is enhanced drastically. In addition the quadratic term $\mu_{0} \mathrm{H}_{\mathrm{kl}}$ is increased by a smaller amount. Both effects indicate a substantial change of the magnetic properties at the interface of the magnetic and the dielectric layer that is caused by a diffusion of nitrogen into the magnetic layer. Furthermore the experiments show that using Kerr Torque Magenetometry this change can be detected very sensitively. 


\section{Kerr hysteresis}
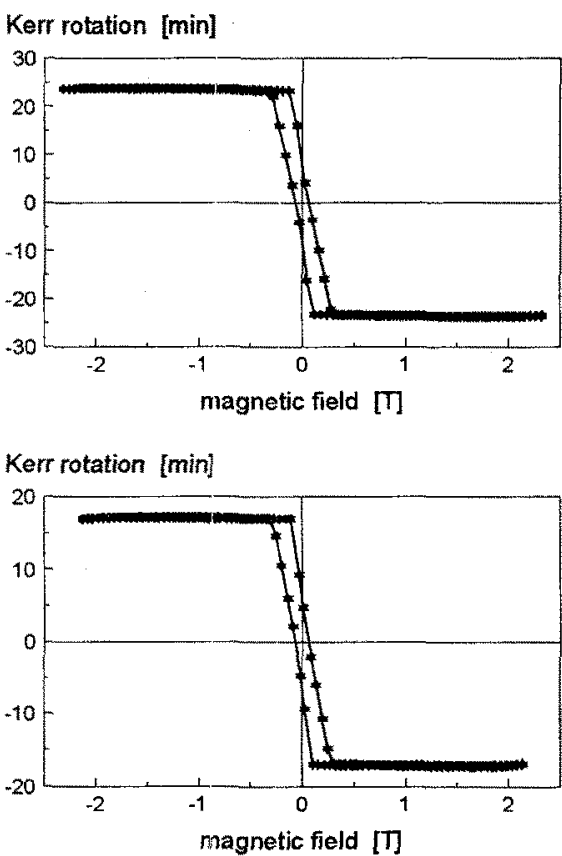

Kerr Torque Magnetometry
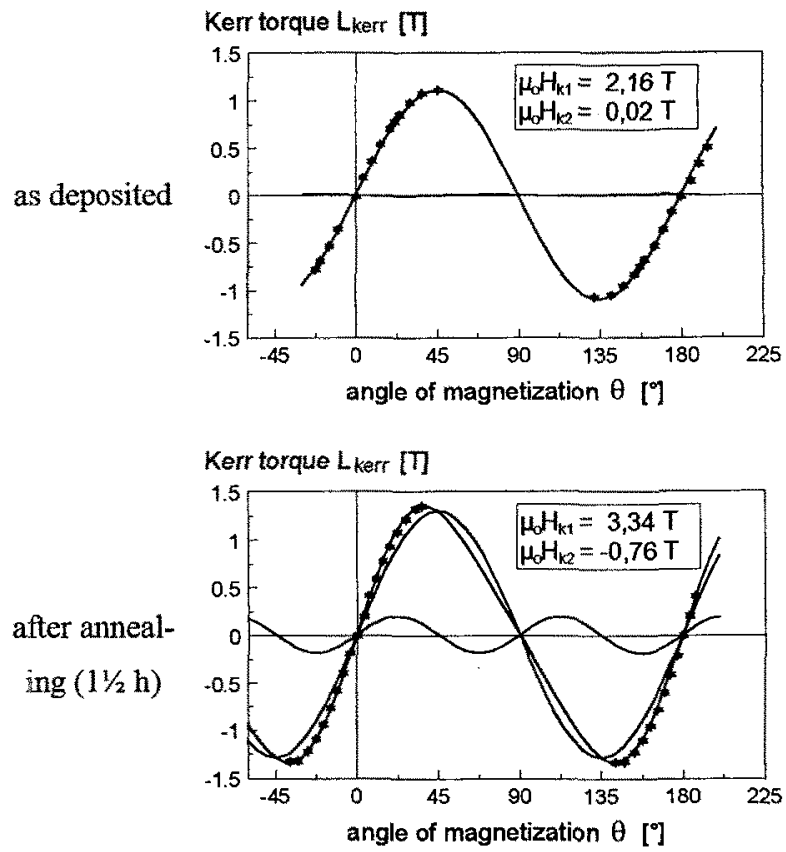

fig.4: Kerr hysteresis and Kerr torque measurements of $\mathrm{T} \mathrm{Tb}_{30} \mathrm{Fe}_{62} \mathrm{Co}_{8}$ sample (500nm) covered by a SiN protective layer sputtered with a surplus of nitrogen

To verify this interpretation samples were prepared, where one of the SiN dielectric layers was separated from the TbFeCo film by a pure Si buffer with a thickness of a few monolayers (fig.5a,b). Additionally samples were prepared with a Si buffer on both sides (fig.5c).

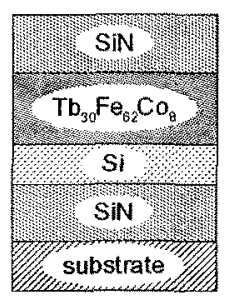

(a)

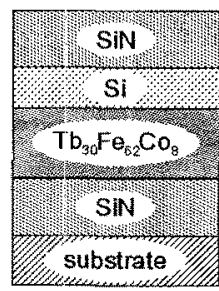

(b)

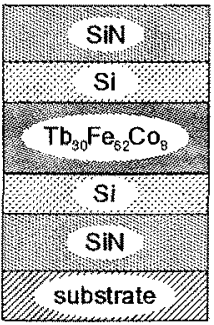

(c) fig. 5: Cross section of the samples with an additional Si buffer between the magnetic and the dielectric layer

For these samples the Kerr hysteresis were measured as a function of temperature from both sides. As an example the results are shown for the first layer stack (fig.5a). At the glass sicle, where the buffer layer was present, a magnetic behaviour was observed without any anomalies (fig.6d-f), i.e. the magnetic layer became paramagnetic above the Curie temperature.

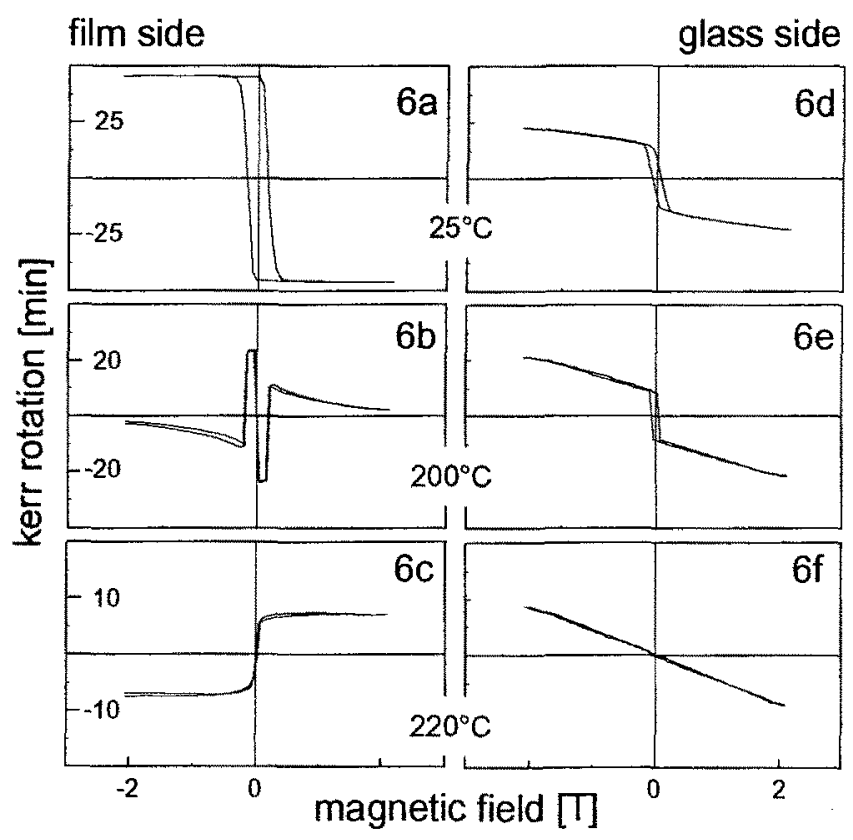

fig. 6: Kerr hysteresis measurements of a $\mathrm{Tb}_{30} \mathrm{Fe}_{62} \mathrm{Co}_{8}$ single layer with a Si buffer layer on the glass side 
Quite in contrast to this the Kerr measurements from the film side are modified substantially (fig.6a-c): At higher temperatures the part of the magnetic layer close to the interface which was magnetically dominated by the RE content at room temperature is dominated by the TM content. Additionally, the Curie temperature of this area is increased. Furthermore, at a temperature of $200^{\circ} \mathrm{C} \mathrm{a}$ complicated shape of the Kerr hysteresis was observed which is typical for an exchange coupled bilayer with a strong coupling energy [9].

Analogous results were observed for the samples having the Si buffer on the other side. In this case also the Kerr hysteresis were modified only at the side where no additional $\mathrm{Si}$ buffer was present separating the $\mathrm{SiN}$ layer from the $\mathrm{TbFeCo}$ film. For the samples with an additional Si intermediate layer on both sides no modifications of the magnetic behaviour could be observed.

The entire results can be explained by assuming a diffusion of nitrogen from the protective layer into the $\mathrm{TbFe}-$ Co film. To exclude a possible oxidation of the magnetic layer a depth profile using Auger spectroscopy was performed additionally. As a result even after annealing for $9 \frac{1}{2} \mathrm{~h}$ no oxygen could be detected in the samples.

The diffusion process leads to a reduced content of the magnetically active $\mathrm{Tb}$ due to a reaction of the magnetic $\mathrm{Tb}$ with the nitrogen. This reaction causes a change of the magnetization in the interface area. Further annealing has proved, that the content of the magnetically active $\mathrm{Tb}$ is continuously reduced. As a consequence a $\mathrm{TM}$ dominated area at the interface between the magnetic film and the protective layer is formed. This leads finally to an antiparallel coupled double layer structure within the magnetic film.

Furthermore the change of the magnetization in the magnetic layer leads to an increasing inhomogeneity of the anisotropy fields within the information depth of the laser beam. This appears in an increasing bi-quadratic term of the anisotropy that can be detected very sensitively by the Kerr Torque Magnetometry already after an annealing time of $1 \frac{1}{2} \mathrm{~h}$.

\section{CONCLUSIONS}

Preparation of SiN dielectric layer with a surplus of nitrogen affects significantly the magnetic properties of sputtered TbFeCo films. This is caused by a diffusion of nitrogen from the protective layer into the magneto-optical layer. The reaction between $\mathrm{Tb}$ and nitrogen changes the content of the magnetically active $\mathrm{Tb}$ within the interface area SiN/TbFeCo and leads in extreme to the formation of an antiparallel coupled double layer.

Therefore, to ensure the long-term stability of MO recording media the reactive sputtering process of the protective layers has to be performed in a way that avoids an influence of nitrogen on the magnetic RE/TM films. Furthermore the Kerr Torque Magnetometry has proved to be a very sensitive tool to detect small changes of physical properties which are correlated to changes of the magnetic anisotropy field.

\section{REFERENCES}

[1] T. C. Anthony, J. Brug, S. Naberhuis and H. Birecki, J. Appl. Phys. 59(1), 213 (1986)

[2] S. Hashimoto et al., IEEE Transactions on Magnetics, Vol. MAG-23 (5), 2278 (Sept. 1987)

[3] X. Qiu and E. Gyarmati, Thin Solid Films 151, 223 (1987)

[4] C.S.Gudeman and D. Mauri, J. Appl. Phys. 67 (9), 5938 (1990)

[5] Te-Ho Wu, Hong Fu, R. A. Hajjar, T. Suzuki and M. Mansuripur,
J. Appl. Phys. 73 (3), 1368 (1993)

[6] H. Hornauer, T. M. Atmono and K. Röll, J. Magn. Magn. Mat. 83, 551 (1990)

[7] H. Rohrmann, M. Kratzer and K. Röll, J. Magn. Magn. Mat. 148, 136 - 138 (1995)

[8] W. Andrä et al., J. Magn. Magn. Mat. 113, 155 (1992)

[9] S. Becker, H. Rohrmann and K. Röll, submitted to J. Magn. Magn. Mat. 